J. Phys. IV France 130 (2005) 63-74

(C) EDP Sciences, Les Ulis

DOI: $10.1051 /$ jp4:2005130004

\title{
Études par diffraction de fibres de I'ADN double brin
}

\author{
V.T. Forsyth ${ }^{1,2}$ et I.M. Parrot ${ }^{1,2}$ \\ 1 Institut Laue Langevin, 6 rue Jules Horowitz, 38042 Grenoble Cedex 9, France \\ 2 Institute of Science and Technology in Medicine, Keele University Medical School, \\ Staffordshire ST4 7QB, UK
}

\begin{abstract}
Résumé L'état fibreux est un état naturel pour les molécules de polymère qui ont tendance à adopter des conformations hélicoïdales régulières plutôt que des structures globulaires caractéristiques à de nombreuses protéines. La diffraction de fibres a donc une application étendue pour l'étude d'une grande variété de polymères biologiques et synthétiques. Ce papier a pour objectif d'illustrer l'étendue générale de la méthode et, en particulier, de démontrer l'impact des sources modernes de rayonnement synchrotron et de faisceaux neutroniques.
\end{abstract}

Table des matières

1 Introduction $\quad 63$

2 Arrangements fibrillaires $\quad 64$

3 Voir les petites choses : de la microscopie optique aux rayons $\mathrm{X}$ et aux neutrons 66

4 Diffraction de l'ADN - Une analogie simple avec la diffraction optique 67

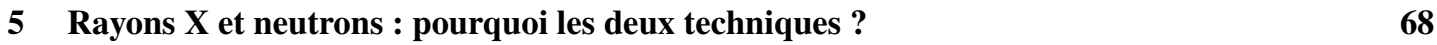

6 Polymorphisme de l'ADN : transitions structurelles $\begin{array}{ll}\text { et importance de l'eau } & \mathbf{7 0}\end{array}$

$\begin{array}{lll}7 & \text { Diffraction de fibres par rayonnement synchrotron } & 70\end{array}$

8 Diffraction des neutrons $\quad 72$

9 Conclusions $\quad 74$

\section{INTRODUCTION}

Une fibre alignée est caractérisée par la présence de nombreuses zones dans lesquelles les molécules de polymère sont disposées avec leurs axes longitudinaux parallèles à l'axe de la fibre. Elles sont typiquement séparées par des régions amorphes moins ordonnées. L'alignement moléculaire dans une telle fibre est associé à différents degrés d'ordre dans l'assemblage côte à côte des chaînes. Dans une large mesure, cet ordre déterminera l'apparence générale du diagramme de diffraction observé qui peut aller d'une variation continue d'intensité à l'autre extrême, où le diagramme est dominé par de fortes réflexions de Bragg. Comme il n'y a pas d'orientation privilégiée des microcristallites autour de l'axe de la fibre, un diagramme de diffraction des rayons $\mathrm{X}$ d'une fibre cristalline a une partie du caractère de ce qui serait obtenu d'un monocristal si on lui faisait effectuer une rotation de $360^{\circ}$ autour de l'un de ses principaux axes au cours de la collecte de données (cf. Figure 1). La résolution obtenue au cours des 


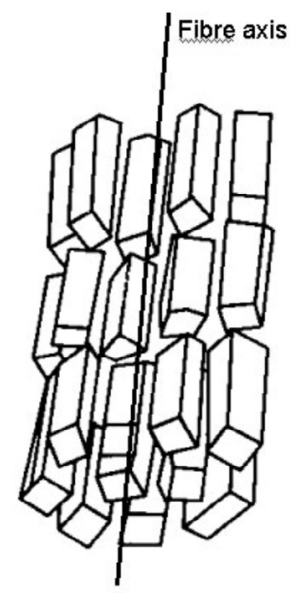

Figure 1. Diagramme illustrant l'orientation aléatoire des cristallites autour de l'axe de la fibre dans le cas d'une fibre cristalline.

expériences de diffraction de fibres varie fortement mais peut, dans des circonstances favorables, aller jusqu'à la résolution atomique. Comme il y a une perte d'information imposée par la moyennisation cylindrique en diffraction de fibres, cela doit être compensé par le fait que, dans la plupart des situations, des mesures comparables ne sont pas possibles à partir de systèmes monocristallins. Dans de nombreuses structures biologiques, comme le muscle et le tissu conjonctif, et également dans les polymères industriels synthétiques, l'état fibreux est important d'un point de vue fonctionnel et la diffraction de fibres fournit la technique la plus puissante pour établir à la fois la structure moléculaire et les interactions d'ordre supérieur de ces molécules. Il est également exact de dire que la diffraction de fibres est une des rares méthodes quantitatives qui est bien adaptée à l'étude des voies conformationnelles dans les molécules de polymère. De telles investigations sont d'une importance centrale dans l'étude du polymorphisme de l'ADN (où des transitions structurales hautement coopératives sont observées) et également dans l'étude des polymères synthétiques où les méthodes d'étirage et de recuit ont un intérêt direct pour les processus industriels.

En raison de l'importance des systèmes hélicoïdaux en diffraction de fibres, la première partie de ce papier est consacré à l'illustration de la diffraction hélicoïdale de l'ADN à l'aide de simples analogies optiques. L'autre partie du papier donne quelques exemples spécifiques qui démontrent l'étendue des méthodes utilisant les rayons $\mathrm{X}$ et les neutrons dans l'étude du polymorphisme de l'ADN.

\section{ARRANGEMENTS FIBRILLAIRES}

Les fibres contiennent des molécules de polymère dont les axes longitudinaux sont généralement parallèles à l'axe de la fibre. Dans le cas d'une fibre cristalline, les molécules de polymère forment de petits cristallites dont un axe est aligné le long de l'axe de la fibre. Comme illustré en Figure 1, ces cristallites n'ont pas d'orientation privilégiée autour de l'axe de la fibre et les données sont par conséquent moyennées sur un cylindre. La nature de la moyennisation dépendra de la symétrie cristalline. Le diagramme de diffraction montre des tâches bien définies d'un bout à l'autre.

Dans le cas d'une fibre non cristalline, les molécules de polymère ne sont pas assemblées en cristallites. Elles sont toujours largement parallèles les unes aux autres bien que leur orientation soit aléatoire. Il n'y a par conséquent pas d'échantillonnage cristallin. Le diagramme de diffraction montre une "diffraction continue" au lieu des fortes réflexions observées pour les fibres cristallines (voir Figure 2). 


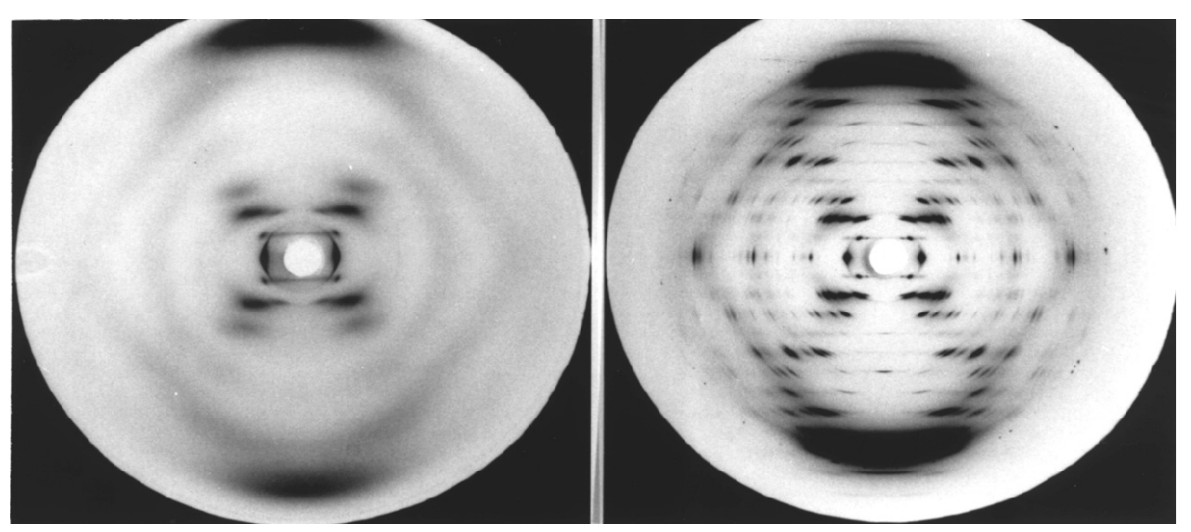

Figure 2. Deux diagrammes de diffraction de fibres d'ADN-B illustrant un échantillonnage cristallin et un échantillonnage non-cristallin. La figure (a) montre une diffraction de fibres aux rayons- $\mathrm{X}$ de la conformation B semi-cristalline, la Figure (b) une diffraction d'une forme B cristalline de l'ADN.

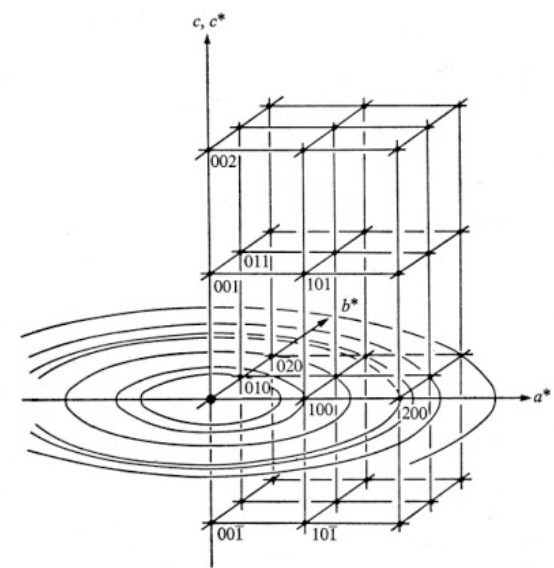

(a)

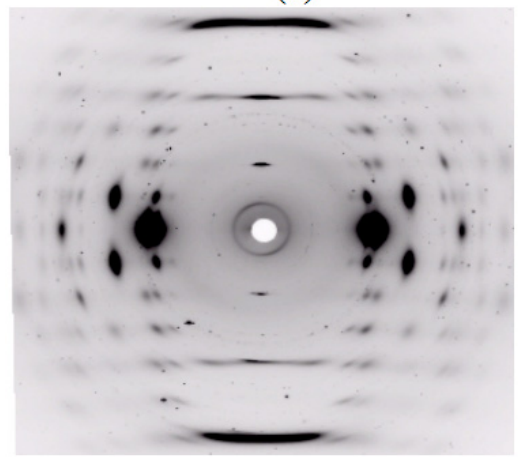

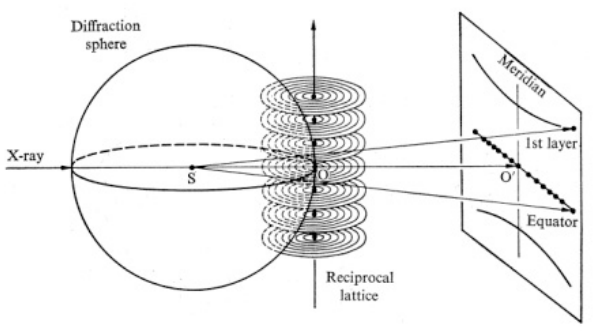

(b)

(c)

Figure 3. Diagrammes montrant comment la moyennisation cylindrique produit un recouvrement des réflexions dans un diagramme de diffraction de fibres cristallines: (a) et (b) montrent comment un cristal en rotation autour de l'axe de la fibre a pour résultat effectif des points du réseau réciproque tracés en "cercles" réticulaires; (c) montre un diagramme de diffraction de fibres cristallines aux rayons X qui illustre cet effet (diagramme de diffraction, Dr Gardner \& DuPont). 
En fonction de la symétrie du cristal, la moyennisation cylindrique aura pour conséquence un recouvrement parfait de différentes classes de réflexions dans un diagramme de diffraction de fibres. On parle de recouvrement systématique. La moyennisation cylindrique introduira également un "recouvrement accidentel" des réflexions qui sont radialement proches l'une de l'autre dans l'espace réciproque. Le recouvrement accidentel s'aggrave pour des angles de diffraction plus élevés.

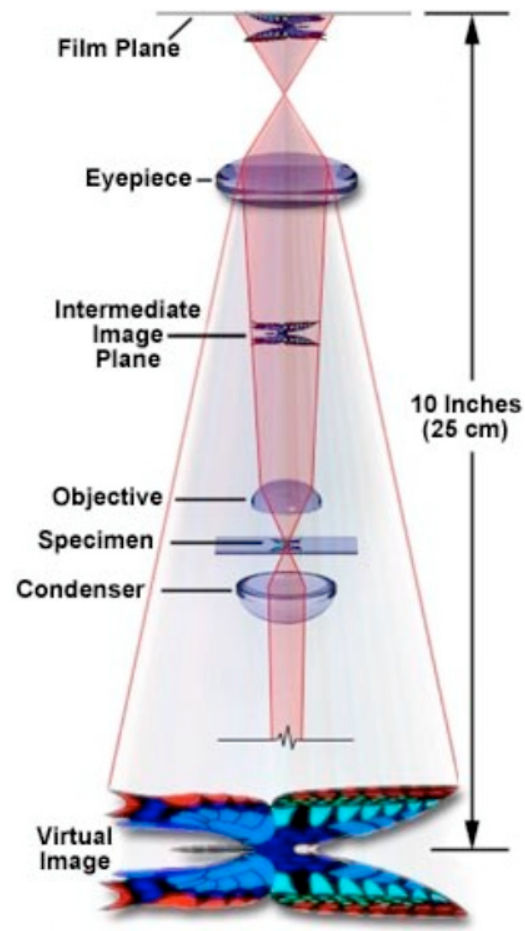

(a)

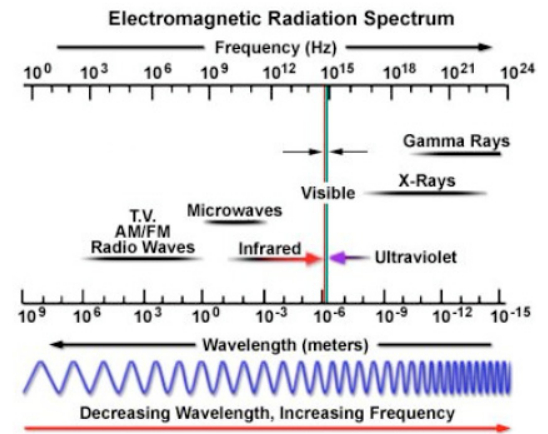

(b)

Figure 4. Un microscope optique peut être utilisé pour avoir une image des objets, il est cependant limité en résolution par la longueur d'onde de la lumière. Pour voir des objets à une échelle plus petite, les rayons X et les neutrons peuvent être utilisés. Cependant, il n'y a pas l'équivalent d'une lentille pour le traitement de l'image - le traitement de l'image doit être effectué de manière analytique.

\section{VOIR LES PETITES CHOSES : DE LA MICROSCOPIE OPTIQUE AUX RAYONS X ET AUX NEUTRONS}

La Figure 4 essaie de résumer comment l'image se forme dans un microscope optique. La lumière incidente arrive sur l'objet et est diffusée par cet objet. La lumière diffusée (diffractée) est recombinée par un système de lentilles pour former une image qui peut être directement interprétée. La résolution disponible en microscopie optique est limitée par la longueur d'onde de la lumière utilisée - en cas de lumière visible, elle est de l'ordre de 400-750 nm (ou 0,40-0,74 $\mu \mathrm{m}$ ). Pour être en mesure de voir les objets avec une meilleure résolution, il faut utiliser des longueurs d'ondes plus petites. Pour voir les structures moléculaires, des longueurs d'onde de l'ordre de 0,1 nm sont nécessaires. Pour les radiations électromagnétiques, cela signifie les rayons X. Les neutrons ont également une longueur d'onde appropriée. Un problème subsiste - il n'existe aucun dispositif disponible pour les rayons $\mathrm{X}$ et les neutrons qui puissent effectivement assumer la fonction des lentilles utilisées en spectroscopie optique. Par conséquent, pour un traitement de l'image à partir des expériences réalisées avec des 


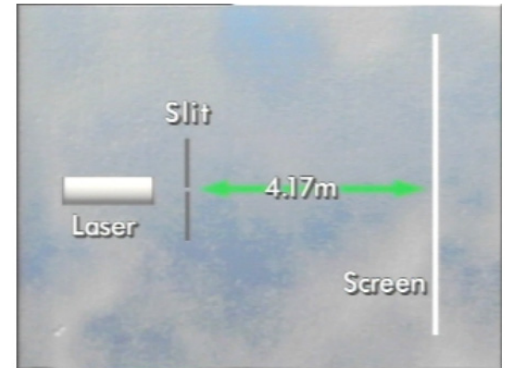

(a)

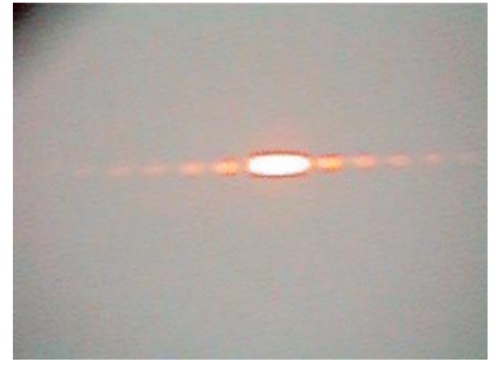

(b)

Figure 5. Diffraction de lumière visible par une ouverture simple. (a) montre l'installation utilisée et (b) le diagramme de diffraction "fente simple" caractéristique observé sur l'écran.

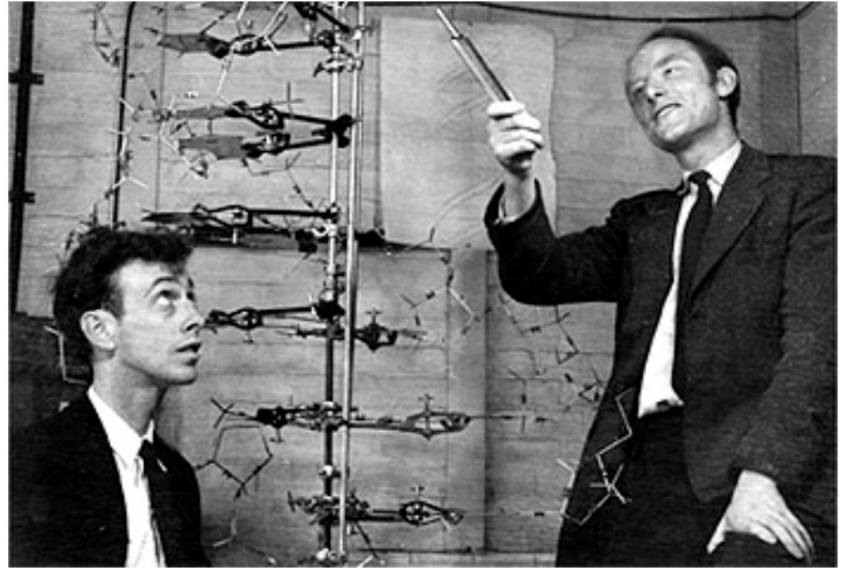

(a)

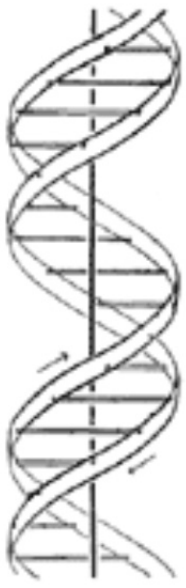

(b)

Figure 6. Modèle Watson \& Crick pour l'ADN. (a) montre leur modèle fabriqué avec des fils métalliques et (b) le diagramme schématique en double hélice comme publié dans Nature 171, 737-738 (1953).

rayons $\mathrm{X}$ et avec des neutrons, le processus de traitement de l'image est généralement effectué par informatique.

Considérez la diffraction de la lumière d'un simple objet - une ouverture simple, comme illustré en Figure 5 - le diagramme de diffraction observé est très simple.

\section{DIFFRACTION DE L'ADN - UNE ANALOGIE SIMPLE AVEC LA DIFFRACTION OPTIQUE}

La Figure 7 montre comment il est possible de considérer la molécule d'ADN comme étant constitué d'un simple arrangement de fentes du type présenté en Figure 5. Elle montre également le type de diagramme de diffraction attendu de chacun de ses objets représenté par une fente.

La Figure 8 montre la conformation B de l'ADN et le diagramme de diffraction enregistré dans une expérience réelle de diffraction de fibres aux rayons $\mathrm{X}$. 

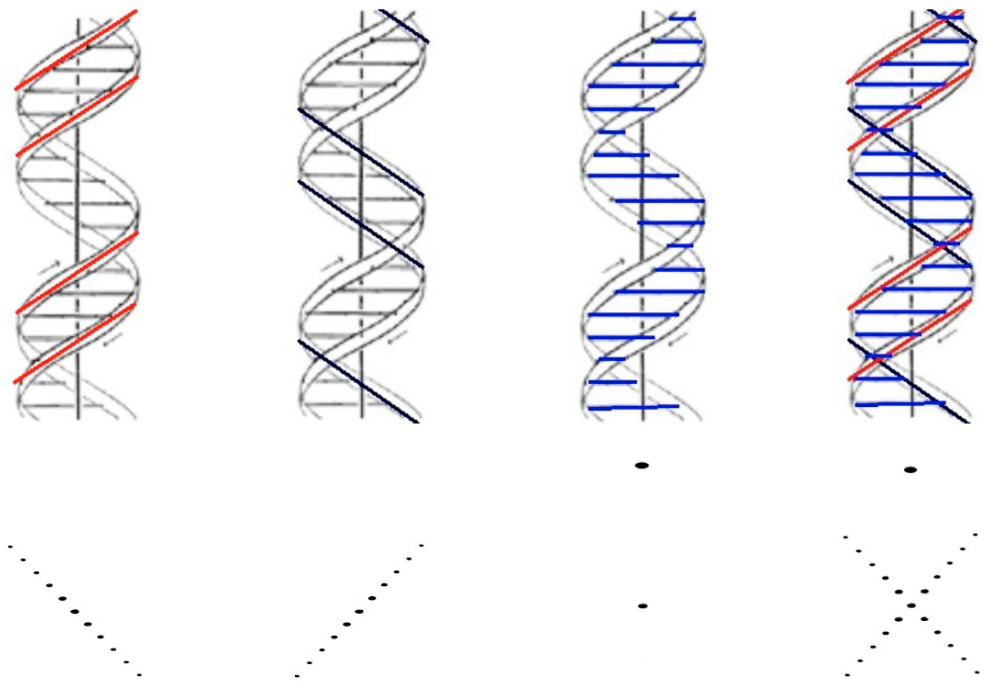

Figure 7. Diagramme montrant (en haut) comment le squelette et la structure des paires de base de l'ADN peuvent être représentés de manière très simplifiée comme une série de fentes simples. Les schémas en bas illustrent les diagrammes de diffraction attendus de chacun des objets situés directement au-dessus.

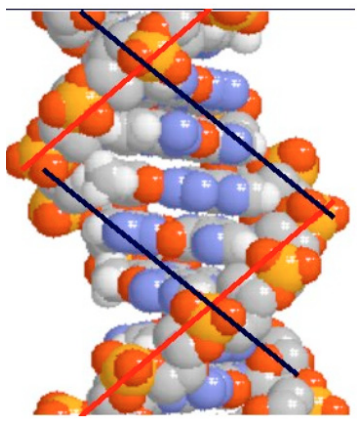

(a)

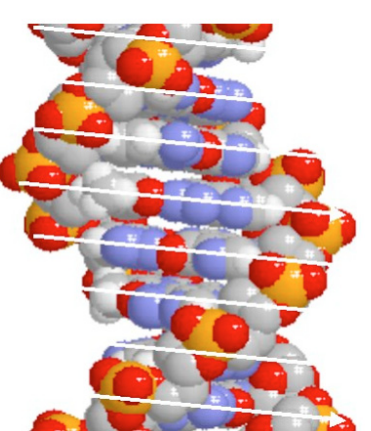

(b)

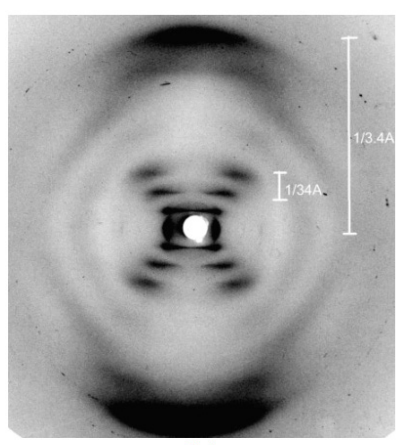

(c)

Figure 8. La structure de l'ADN-B semi-cristallin et le diagramme de diffraction de fibres enregistré. (a) et (b) montrent la relation entre le modèle moléculaire et les systèmes de fentes simples décrits à la Figure 7 . (c) montre le diagramme de diffraction des rayons X, qui peut être comparé avec les schémas du bas de la Figure 7.

\section{RAYONS X ET NEUTRONS : POURQUOI LES DEUX TECHNIQUES ?}

La Figure 9 montre le polygone scientifique de Grenoble où sont implantées sur un site commun des installations scientifiques majeures incluant la source de neutrons de l'Institut Laue-Langevin (ILL), le Laboratoire Européen de Rayonnement Synchrotron (ESRF) et le Laboratoire Européen de Biologie Moléculaire (EMBL). L'Institut de Biologie Structurale (IBS) qui dispose d'appareillages RMN de forte puissance est situé à proximité. Ce site offre les meilleures installations actuelles au monde pour la diffraction avec les neutrons et les rayons X. L'ESRF fournit des faisceaux de rayons X d'une très grande 


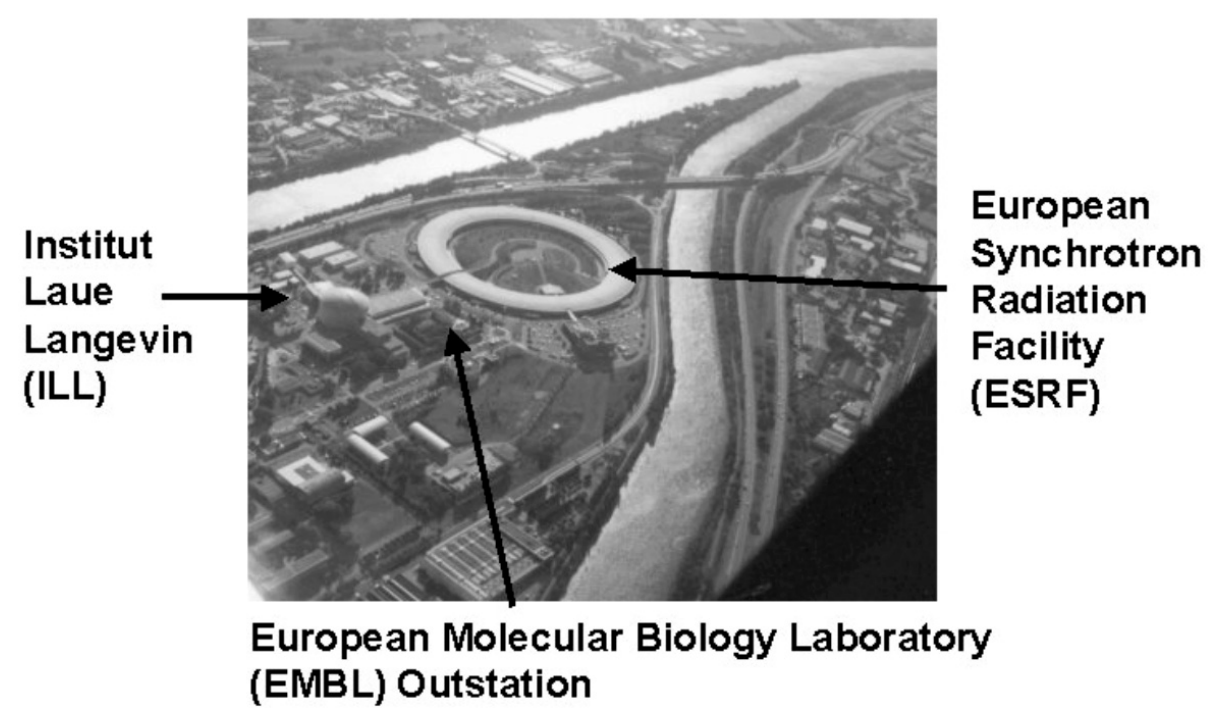

Figure 9. Photo du polygone scientifique de Grenoble montrant l'ILL, l'ESRF et l'EMBL.

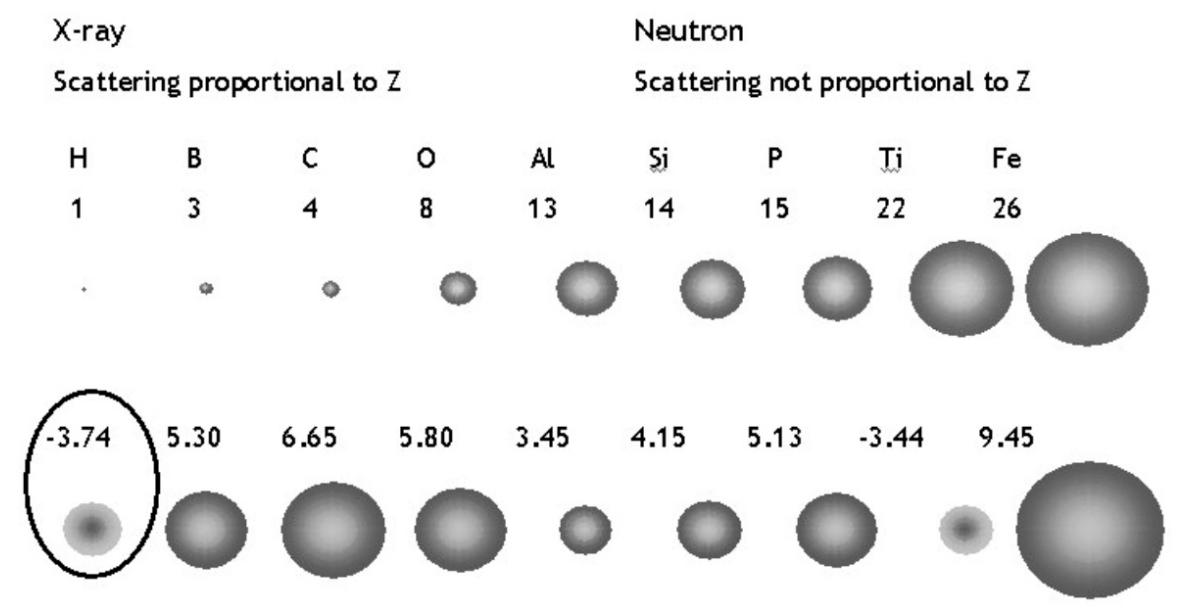

Figure 10. Diagramme pour illustrer les forces de diffraction d'un certain nombre d'éléments pour les neutrons et les rayons $X$. La rangée supérieure de sphères représente l'augmentation de la diffraction des rayons $\mathrm{X}$ avec Z. La rangée du bas illustre la variation pour la diffraction neutronique. Le fait que l'hydrogène (entouré dans le diagramme) puisse être remplacé par son isotope, le deutérium, qui a une force de diffusion neutronique beaucoup plus importante, présente un intérêt particulier pour l'étude des systèmes biologiques.

intensité qui peuvent être utilisés pour enregistrer des diagrammes de diffraction à partir de systèmes qui varient dans le temps ou à partir d'échantillons qui sont trop petits pour être étudiés avec une source normale de rayons X. L'ILL est doté d'un réacteur à Haut Flux, la source de neutrons la plus puissante au monde, qui est bien adapté à l'étude de l'hydrogène ou de l'eau dans les systèmes biologiques (ce qui est très difficile et parfois impossible à réaliser avec les rayons X). La Figure 10 illustre pourquoi les rayons $\mathrm{X}$ et les neutrons sont des outils hautement complémentaires. 


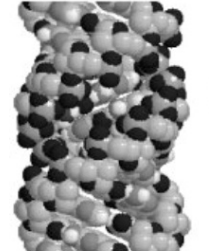

A-DNA

$\mathrm{RH}$

$11 \mathrm{bp} /$ turn

pitch $=28.2 \mathrm{~A}$

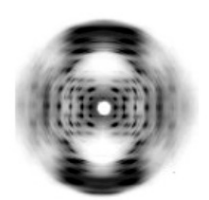

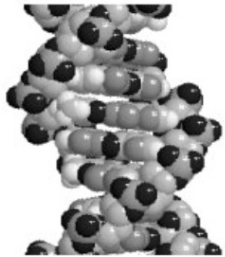

B-DNA

$\mathrm{RH}$

$10 \mathrm{bp} / \mathrm{turn}$

pitch=34A

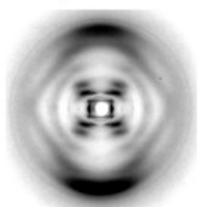

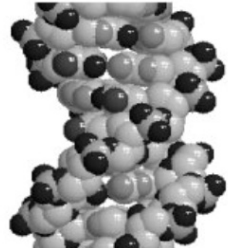

C-DNA $\mathrm{RH}$

9.33 bp/tum pitch=31A

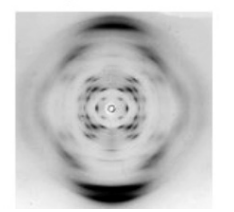

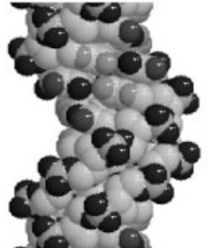

D-DNA

$\mathrm{RH}$

$8 \mathrm{bp} /$ turn pitch $=24.2 \mathrm{~A}$

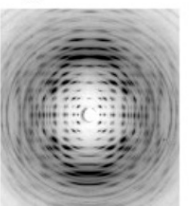

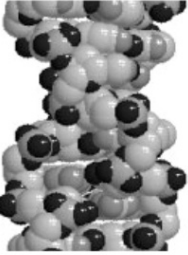

Z-DNA

$\mathrm{LH}$

12 bo/turn

pitch $=43 \mathrm{~A}$

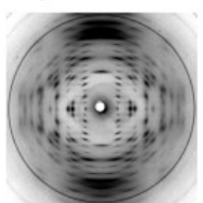

Figure 11. Les 5 conformations principales adoptées par l'ADN double brin et les diagrammes de diffraction des rayons $\mathrm{X}$ dont leurs structures sont dérivées.

\section{POLYMORPHISME DE L'ADN : TRANSITIONS STRUCTURELLES ET IMPORTANCE DE L'EAU}

Les principales conformations de la double hélice d'ADN sont illustrées en Figure 11, avec leurs diagrammes de diffraction aux rayons $\mathrm{X}$ respectifs. Ces conformations peuvent être adoptées par la double hélice d'ADN en fonction de trois paramètres : l'hydratation du milieu, les conditions ioniques et la séquence nucléotidique (voir Figure 12). Les Figures 11 et 12 illustrent, premièrement, le fait que l'ADN double brin est capable d'une flexibilité considérable dans sa structure tridimensionnelle et, deuxièmement, que les transitions entre ces structures peuvent apparaître en fonction du contexte aqueuxce qui est relativement facile à réaliser en ajustant l'humidité de l'environnement de la fibre. Certains changements stéréochimiques impliqués dans ces transitions sont assez mystérieux - par exemple le changement entre les formes A et B de l'ADN - ADN dont le pas l'hélice est à droite-, ou encore celui entre les formes $\mathrm{Z}$ et $\mathrm{B}$ de l' $\mathrm{ADN}$ - la forme $\mathrm{Z}$ étant particulière puisque le pas de l'hélice est à gauche.

La disponibilité de sources de rayonnement synchrotron depuis le début des années 80 a considérablement étendu la puissance des techniques de diffraction de fibres. En particulier, en utilisant une installation comme l'ESRF en parallèle avec la technologie moderne des détecteurs, il est possible d'enregistrer un diagramme de diffraction d'une fibre d'ADN d'un diamètre de 100 microns en moins d'une seconde au lieu des heures et parfois des jours qui étaient nécessaires avec une source de rayons $\mathrm{X}$ conventionnelle. Il est ainsi possible de suivre les transitions structurelles en temps réel et d'analyser les données et ainsi obtenir une carte détaillée des voies stéréochimiques suivies au cours de ces transitions.

\section{DIFFRACTION DE FIBRES PAR RAYONNEMENT SYNCHROTRON}

La Figure 13 donne un bon exemple d'une transition conformationelle en fonction de l'hydratation dans l'ADN double brin de poly[d(A-T)].poly [d(A-T)]. Comme indiqué dans la seconde case de la figure 12, il s'agit d'un simple polymère répétitif contenant des bases A-T qui alternent le long de chaque brin. La Figure 13 montre des données de diffraction de fibres aux rayons $\mathrm{X}$ résolues dans le temps et enregistrées pour la transition entre les formes $\mathrm{D}$ et B de l'ADN - une transition qui implique une ouverture de l'hélice qui passe d'une hélice formée de 8 paires de bases par tour avec un pas de $\sim 24 \AA$ à une hélice formée de 10 paires de bases par tour avec un pas de $\sim 34 \AA$. 


\begin{tabular}{|c|c|c|c|c|}
\hline \multirow{10}{*}{$\begin{array}{l}\frac{\text { NATURAL DNA }}{\text { "Random" sequence }} \\
\mathrm{C} \leftrightarrow \mathrm{A} \leftrightarrow \mathrm{B}\end{array}$} & $\frac{\text { ALTERNATING }}{\underline{\text { A-T }}}$ & $\frac{\text { ALTERNATING }}{\underline{\text { G-C }}}$ & $\frac{\text { HOMOPOLYMER }}{\text { G-C }}$ & $\frac{\text { HOMOPOLYMER }}{\underline{\text { A-T }}}$ \\
\hline & $\uparrow \mathrm{A}-\mathrm{T}$ & $\uparrow \mathrm{G}-\mathrm{C}$ & $\uparrow \mathrm{G}-\mathrm{C}$ & $\uparrow \mathrm{A}-\mathrm{T}$ \\
\hline & T - A & $\mathrm{C}-\mathrm{G}$ & G-C & A - T \\
\hline & T - A & $\mathrm{C}-\mathrm{G}$ & G - C & A- T \\
\hline & A- T & $\mathrm{G}-\mathrm{C}$ & G-C & A - T \\
\hline & $\mathrm{T}-\mathrm{A}$ & $\mathrm{C}-\mathrm{G}$ & $\mathrm{G}-\mathrm{C}$ & A - T \\
\hline & A- T & $\mathrm{G}-\mathrm{C}$ & G-C & $A-T$ \\
\hline & $\mathrm{T}-\mathrm{A}$ & $\mathrm{C}-\mathrm{G}$ & G- C & A- $T$ \\
\hline & \begin{tabular}{|l} 
A- T \\
T - A
\end{tabular} & $\mid \begin{array}{l}\mathrm{G}-\mathrm{C} \\
\mathrm{C}-\mathrm{G}\end{array}$ & $\begin{array}{l}G-C \\
G-C\end{array}$ & $\begin{array}{l}A-T \\
A-T\end{array}$ \\
\hline & $D \leftrightarrow B$ & $Z \leftrightarrow B$ & A & B \\
\hline
\end{tabular}

Figure 12. Différentes séquences d'ADN naturel et synthétique et les structures adoptées en fonction de l'hydratation.
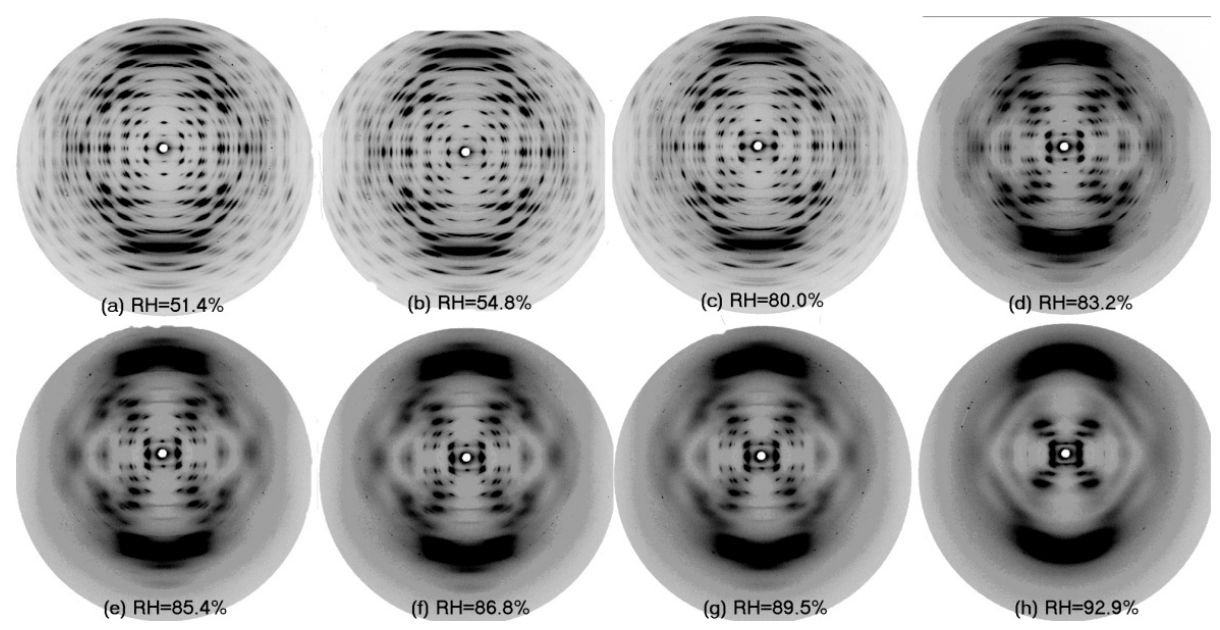

Figure 13. Diagrammes de diffraction de fibres obtenus par rayonnement synchrotron enregistrés pendant la transition induite par l'hydratation entre les formes D et B de l'ADN.

Cette étude montre que la transition se fait par une ouverture en douceur de l'hélice pendant laquelle le pas varie en continu entre $24 \AA$ et $34 \AA$.

La Figure 14 montre un autre bon exemple - une étude réalisée à l'ESRF de la transition A-B dans l'ADN naturel d' Escherichia coli.

Ce type d'expérience avait été réalisé précédemment sur une source de 2ème génération (avec une résolution en temps de 4-6 minutes) et il n'avait jamais été possible d'observer autre chose qu' un mélange statistique des conformations A et B - c'est-à-dire qu'aucune structure intermédiaire n'avait été observée. La résolution en temps disponible à l'ESRF a montré pour la première fois que cette transition se faisait en fait par des structures intermédiaires détectables mais qu'elle avait un caractère beaucoup plus rapide que d'autres qui avaient été étudiées précédement (comme la transition D-B décrite plus haut). Ceci est illustré sur la Figure 15 qui montre la variation du pas de l'hélice pendant la transition. La partie principale de la transition pendant laquelle la variation du pas de l'hélice est la plus importante a lieu sur une période de $\sim 15$ secondes. La raison pour laquelle cette transition structurelle n'a pas pu être correctement observée sur des sources synchrotron de seconde génération est immédiatement évidente. 

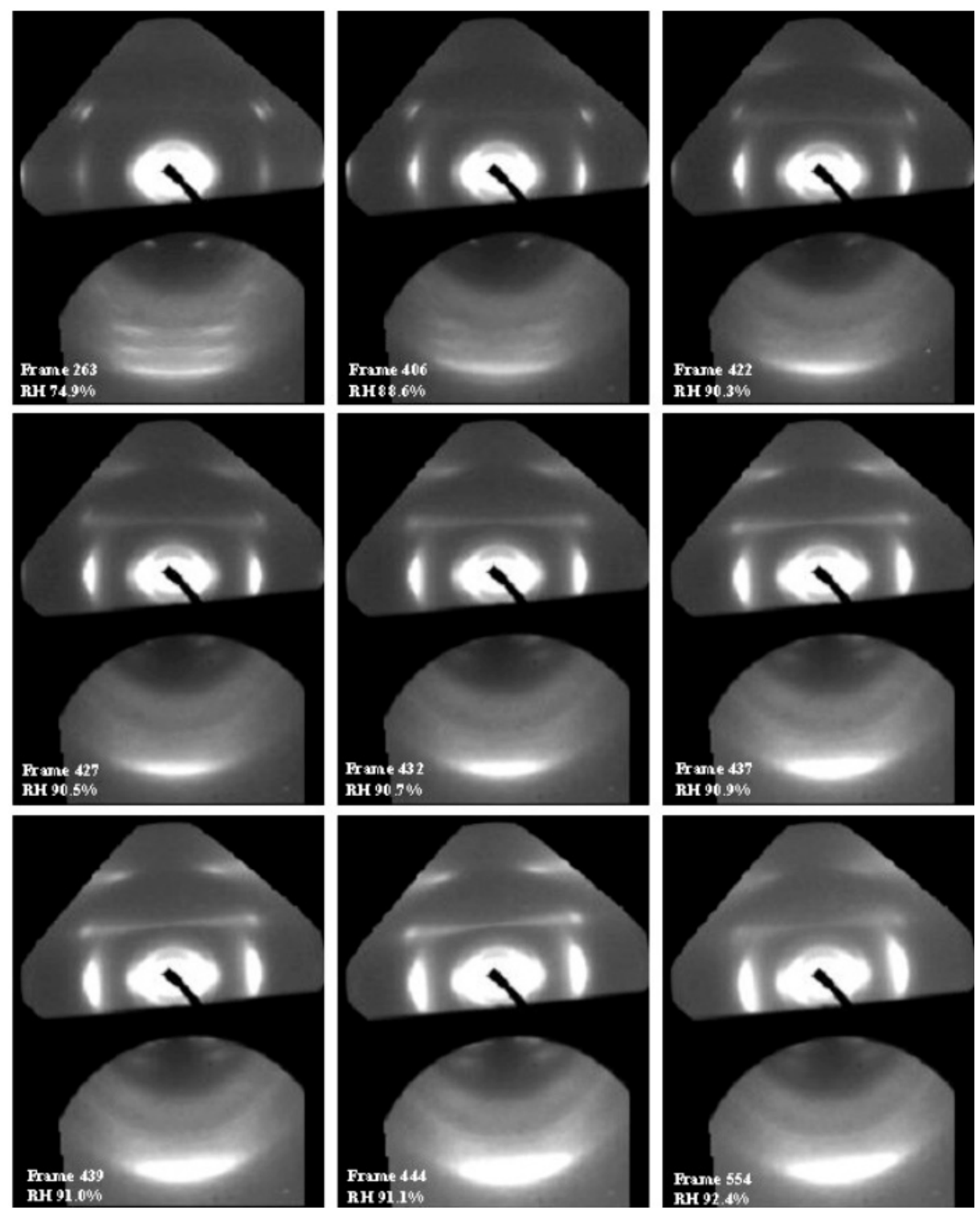

Figure 14. Diagrammes de diffraction de fibres obtenus par rayonnement synchrotron d'une étude en temps résolu de la transition de la forme $\mathrm{A}$ à la forme $\mathrm{B}$ dans l'ADN naturel de $E$. Coli. Cette étude réalisée sur la ligne de faisceau ID02 à l'ESRF, avait une résolution en temps de 0,6 secondes et a montré pour la première fois que la transition impliquait un changement rapide et continu lors de la transition de la forme A à la forme B de l'ADN.

\section{DIFFRACTION DES NEUTRONS}

Les résultats décrits dans les sections précédentes mettent en évidence l'importance de l'eau dans la stabilisation des conformations de l'ADN et dans la médiation des transitions entre ces conformations. Ce fait est reconnu depuis de nombreuses années mais la question de la localisation de l'eau autour de l'ADN n'avait jamais été abordée de manière satisfaisante en utilisant les rayons X. Lors de la détermination de la structure des conformations A et B de l'ADN, l'eau entourant l'ADN a été traitée comme un continuum (réf Langridge et al 1960a and b; Fuller et al 1965). Des tentatives ont été faites 


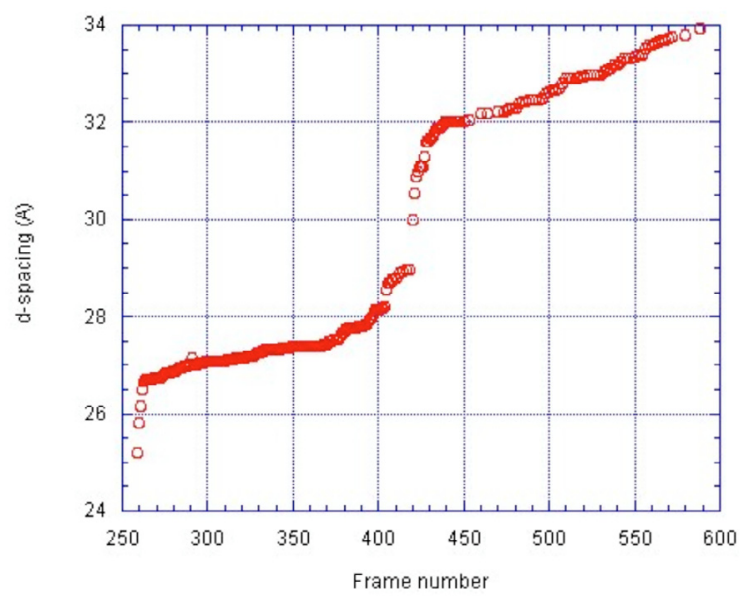

Figure 15. Variation du pas de l'hélice au cours de la transition de la forme A à la forme B de l'ADN. La partie cruciale de la transition a lieu entre les clichés 410 et 435 sur une période de $\sim 15$ secondes.

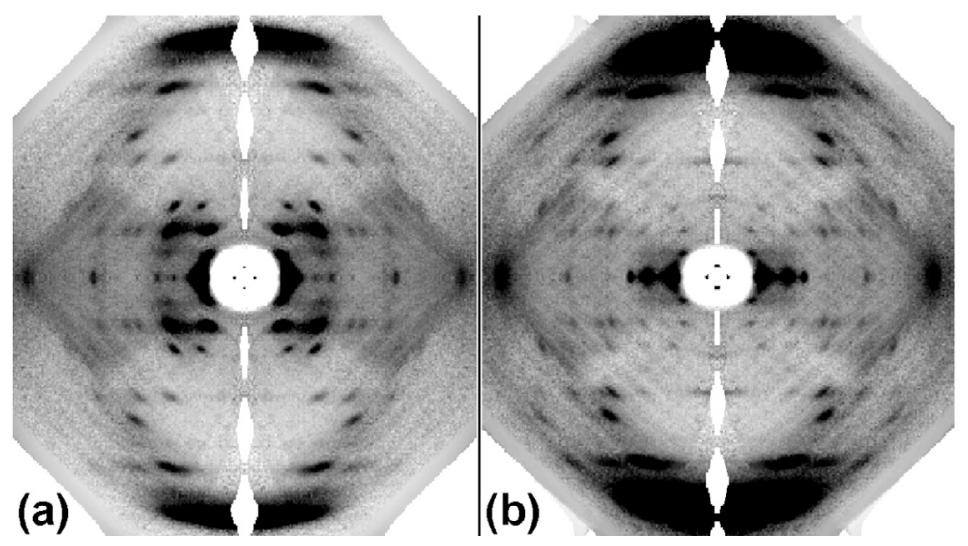

Figure 16. Diagrammes de diffraction de fibres enregistrés avec les neutrons à partir d'ADN (a) hydraté avec $\mathrm{H}_{2} \mathrm{O}$ et (b) hydraté avec $\mathrm{D}_{2} \mathrm{O}$. Ce remplacement isotopique induit des différences d'intensité sur l'ensemble du diagramme de diffraction. Ces différences peuvent être utilisées pour représenter la structure d'hydratation autour de l'ADN.

par Marvin, en utilisant la technique de différence de Fourier, pour localiser les molécules d'eau qui auraient pu être dans des positions fixes à proximité de l'ADN. Cependant, les résultats n'ont pas été convaincants (Marvin, 1960). La disponibilité de faisceaux de neutrons intenses comme ceux de l'ILL a offert la possibilité de localiser l'eau entourant l'ADN en utilisant les techniques par différence de Fourier qui exploitaient le pouvoir de diffusion relativement élevé des neutrons par l'hydrogène et, de manière encore plus significative, en exploitant le remplacement isotopique de l'hydrogène par le deutérium. Cette étude fût réalisée à l'ILL en utilisant des techniques établies pour contrôler l'humidité relative de l'environnement de la fibre et, par conséquent, le degré d'hydratation de l'ADN, pour remplacer $\mathrm{H}_{2} \mathrm{O}$ entourant l' $\mathrm{ADN}$ par $\mathrm{D}_{2} \mathrm{O}$. La Figure 6 montre l'effet du remplacement de $\mathrm{H}_{2} \mathrm{O}$ par $\mathrm{D}_{2} \mathrm{O}$ sur le diagramme de diffraction de fibres enregistré par diffraction de neutrons. La Figure 17 montre quelques exemples des images de différences de Fourier obtenues lors de ces études. 


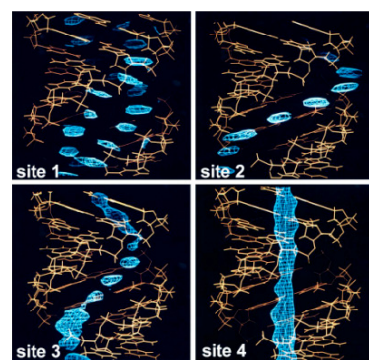

(a)

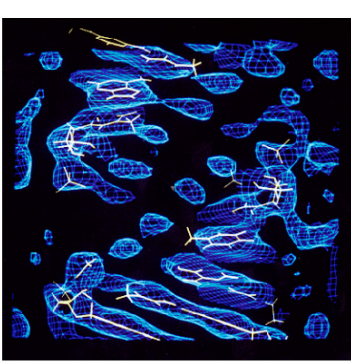

(b)

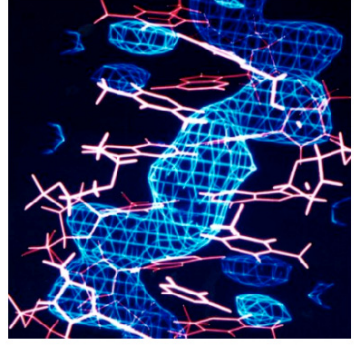

(c)

Figure 17. Images par différence de Fourier montrant (a) quatre régions d'hydratation situées autour de l'ADN-A; (b) une carte de Fourier $2 \mathrm{Fo}-\mathrm{Fc}$ montrant clairement les régions contenant de l'eau reliant les atomes d'oxygène $\mathrm{O} 1$ et de phosphate dans l'ADN-A et (c) l'hydratation dans le sillon mineur de l'ADN.

\section{CONCLUSIONS}

La diffraction de fibres, grâce à la flexibilité structurelle qu'elle permet, est une technique puissante pour identifier les conditions qui favorisent des conformations particulières de l'ADN double brin et qui sont responsables de l'induction de transitions entre elles. Bien que des distorsions graduelles de conformations particulières aient été observées, les conformations de l'ADN double brin sont généralement bien définies, les structures finales après les transitions étant hautement reproductibles.

Outre le fait qu'elle permet de démontrer le caractère induit par l'hydratation de ces transitions, la diffraction de fibres permet également d'élucider le rôle des ions en association avec l'eau. Il est cependant important de souligner que, dans la mesure où les données de diffraction à partir de fibres d'ADN sont typiquement limitées à une résolution de $2,5 \AA$ A, elles ne peuvent généralement pas donner d'informations structurelles à la résolution atomique. L'information à ce niveau, tout au moins en ce qui concerne les atomes autres que l'hydrogène, ont habituellement été obtenus au cours des deux dernières décennies à partir de la détermination des structures de monocristaux d'oligonucléotides. Les études de diffraction de fibres des différentes conformations de l'ADN et l'études des monocristaux d'oligonucléotides peuvent être considérées comme largement complémentaires. Bien que la détermination des structures des monocristaux puissent fournir des informations détaillées sur les positions atomiques, elle présente des limites car la courte longueur de l'oligonucléotide peut être affectée par des "effets de fin de chaînes" et par les forces d'empilement du cristal qui peuvent entraîner des distorsions conformationnelles locales. En outre, contrairement à la situation au sein des fibres d'ADN, l'environnement des monocristaux permet rarement d'avoir des transitions conformationnelles. 